\title{
Effect of HCG-triggered ovulation on pregnancy outcomes in intrauterine insemination: an analysis of 5610 first IUI natural cycles with donor sperm in China
}

\author{
jipeng wan ${ }^{1}$, Zhen-Jing Wang ${ }^{2}$, Yan Sheng ${ }^{2}$, Wei Chen ${ }^{2}$, Qing-Qing Guo ${ }^{2}$, Jin Xu \\ Hua-Rui Fan' ${ }^{2}$, and Mei Sun ${ }^{2}$ \\ ${ }^{1}$ Shandong Provincial Hospital \\ ${ }^{2}$ Affiliation not available
}

May 5, 2020

\begin{abstract}
Objective: To evaluate the effect of human chorionic gonadotropin (hCG) trigger ovulation on pregnancy outcomes in natural IUI cycles with donor sperm. Design: Retrospective cohort study. Setting: Not applicable. Patients: A total 5610 firstnatural IUI cycles with donor sperm in infertile couples during the period from January 2012 to December 2017. To control for other confounding factors, our analysis was restricted to normo-ovulatory women without tubal infertility. Intervention(s): hCG-triggered ovulation Main Outcome Measures: The main outcome measure was live birth rate; the secondary outcomes included rates of clinical pregnancy and miscarriage. Results: In the crude analysis, both the clinical pregnancy $(27.40 \%$ versus $22.73 \%$; $\mathrm{P}=0.001)$ and live birth rates $(24.52 \%$ versus $20.13 \%$; $\mathrm{P}=0.007)$ were significantly higher for the hCG group than for the spontaneous LH group. After adjustment for a number of confounding factors, the reproductive outcomes were still significantly worse for the spontaneous ovulatory group. Conclusions: Among women undergoing natural cycle IUI with donor sperm, hCG triggered ovulation for timing insemination offers beneficial impacts on both clinical pregnancy rates and live birth rates.
\end{abstract}

\section{Introduction}

Intrauterine insemination (IUI), a minimally invasive and low-cost procedure, is a first-line treatment for a broad range of indications in reproductive medicine ${ }^{1,2}$. IUI involves timely scheduled insemination of sperm into the uterus, either in natural cycles or following ovarian stimulation. Compared to stimulated IUI, natural cycle IUI is preferable for patients because of advantages including lower medication cost and reduced rates of multiple gestation ${ }^{3}$.

It is known that the timing of insemination is one of the most impactful factors influencing the success rates of IUI ${ }^{4,5}$. There are various methods for timing IUI, and administration of human chorionic gonadotropin (hCG) is a widely accepted in clinical practice. Administration of hCG requires less endocrine monitoring, but its effect on endometrial receptivity has drawn extensive attention recently ${ }^{6-}{ }^{8}$.

Multiple clinical studies have assessed the effect of hCG on pregnancy outcomes, but have reached inconsistent conclusions $^{9-12}$. Such conflicting results result from clinical indication, female age, baseline characteristics of study participants, sperm quality, timing, or type of ovarian stimulation.

The current study examined a large cohort of women undergoing natural cycle IUI and aimed to assess the effect of hCG-induced ovulation on pregnancy outcome of natural cycles IUI. Attempting to control for sperm-related factors, this retrospective study only examined patients using donor sperm. 


\section{Materials and Methods}

\section{Study Design}

This retrospective study included women who underwent IUI with donor sperm in a natural menstrual cycle. The trial was conducted between January 2012 and December 2017 at the Center for Reproductive Medicine of the Shandong Provincial Hospital Affiliated to Shandong University. The Institutional Review Board approved the research project.

Prior to the IUI cycles, all women underwent basic fertility investigation that included evaluations of the following: cycle day $3 \mathrm{FSH}$, LH, estradiol, anti-Müllerian hormone (AMH), prolactin, and thyroid stimulating hormone (TSH) levels, hysterosonography (at least one month prior to IUI), as well as transvaginal ultrasound for evaluation of pelvic anatomy and follicular monitoring.

Study inclusion criteria were: age [?] 35 years, regular menstrual cycles (28-35 days), no personal history of infertility or medical conditions likely to cause infertility, a normal baseline hormonal profile, and patency of at least one fallopian tube. Exclusion criteria were the presence of irregular menstrual cycles, tubal factor infertility, confirmed endometriosis, a diagnosis of polycystic ovarian syndrome (according to the Rotterdam criteria; Rotterdam ESHRE/ASRM-Sponsored PCOS Consensus Workshop Group, 2004), abnormal TSH or prolactin levels, or any known metabolic or endocrinological disease. Women with incomplete medical records were excluded from the study, as were women who taking any medication.

The choice to use hCG trigger or urinary LH monitoring plus transvaginal ultrasonography was made according to the patient's preference and availability for monitoring. A home urinary ovulation prediction kit was used to monitor urinary LH levels once a day, in the evening around 19:00 h, starting on cycle day 10. And serial ultrasonography for follicular monitoring was also started on cycle day 10 for all cycles. When ovulation was diagnosed, insemination was performed immediately.

In the spontaneous ovulation group (based on urinary LH monitoring and transvaginal ultrasonography), insemination was carried out within 24 hours after detection of the first urinary LH positive test or upon detection of ultrasound features suggestive of ovulation. In the induced ovulation group (administration of hCG trigger), when a leading follicle reached a diameter of $16 \mathrm{~mm}$ or urinary LH surge appeared, ovulation was triggered using 10,000 IU of recombinant hCG (choriogonadotropin alfa, Ovidrel(r); EMD Serono, Inc.). In our practice, the standard of care is to perform two inseminations in a given cycle, approximately 24 hours and 36 hours after HCG injection ${ }^{13-15}$; however, there are clinical factors affecting the clinical decision to use single or double IUI including patient demand, financial barriers, or ovulation time.

Frozen donor sperm were obtained from the Shandong Province Human Sperm Bank of China, and were thawed on site on the day of insemination. All sperm samples were prepared with a density gradient centrifugation method using Puresperm TM (Nidacon, International AB) ${ }^{16}$. The quality of semen was evaluated before and after processing (semen TMS).

The insemination procedure was the same for all women, bed rest was maintained for 15 min after the insemination ${ }^{17}$. A urine pregnancy test was administered 14 days after insemination, and if positive, a transvaginal ultrasound would be performed 14 days later to assess the pregnancy outcome (including the number of gestational sacs, viability, and location).

\section{Data Analyses}

Clinical pregnancies were confirmed by the presence of a fetal heartbeat on ultrasound scanning 7 weeks after insemination. Miscarriage was defined as loss of a clinical pregnancy before the 28th week of gestation. Live birth was defined as delivery of a live neonate after 28 weeks' gestation. Our primary outcome measure was live birth rate. Our secondary outcomes included clinical pregnancy rate and miscarriage rate. In light of the significant differences detected related to intrauterine insemination number between the hCG group and the urinary LH groups, a subgroup analysis was performed to assess the impacts of one vs. two inseminations in a given cycle for both the hCG and urinary LH groups. 
Statistical analysis was performed using SPSS Statistics 20.0 (IBM). Mean values and standard deviations were calculated for each continuous variable, whereas percentages were determined for the categorical variables. Continuous variables were compared with Student'st -tests. Categorical variables were compared with chi-squared and Fisher's exact tests. A single, multivariate logistic regression analysis (incorporating ovulation timing method as a categorical explanatory variable) was performed to assess the independent effect of hCG administration on reproductive outcomes after adjustment for possible confounding factors, including maternal age, BMI, number of intrauterine insemination events, and basal hormone levels (FSH, LH, estradiol, AMH). A $P$ value $<0.05$ was considered statistically significant.

\section{Results}

A total of 5610 patients who were treated with IUI from January 2012 until December 2017 were included in the study. Despite the fact that some women received several IUI treatments per fertility cycle, only data for the first treatment (either single or double insemination) of each woman was included in the analysis.

The baseline characteristics and hormonal profiles of the patients in the hCG and spontaneous ovulation group are presented in Table 1. No significant differences were observed regarding BMI, basal AMH, or estradiol concentration between patients of the two groups, while slight differences were detected in age and basal FSH and basal LH concentrations.

Pregnancy outcomes are shown in Table 2. In crude analysis, compared to the spontaneous ovulation group, the hCG group had significantly higher rates of clinical pregnancy (27.40\% versus $22.73 \%$, OR (95\% CI): 1.28 $(1.11-1.49) ; P=0.001)$ and live birth rates $(24.52 \%$ versus $20.13 \%$, OR (95\% CI); 1.29 (1.11-1.50); $P=0.001)$. Additionally, no difference in the rate of miscarriage was observed between the two groups $(10.50 \%$ versus $11.46 \%$, OR (95\% CI): 0.91(0.60-1.36); $P=0.671)$. Note that the significantly increased clinical pregnancy and live birth rates of the hCG group were still detected after adjustment for a number of confounding factors (Table 2).

To assess the effect of intrauterine insemination number on pregnancy outcomes, a subgroup analysis (single IUI, double IUI) was performed (Table 3,4). Among women who received double IUI, there was no difference between the hCG group and spontaneous ovulation group in the rates of clinical pregnancy (27.30\% VS 25.00\%, OR (95\% CI): $1.12(0.88-1.44) ; P=0.343)$ or live birth (24.46\%VS20.66\%, OR (95\% CI): 1.24 $(0.96-1.62) ; P=0.102)$. However, among single IUI patients, the hCG group women achieved significantly higher rates of clinical pregnancy (28.23\% versus $21.80 \%$, OR (95\% CI): $1.41(1.09-1.81) ; P=0.009)$ and live birth (25.00\% versus $19.91 \%$, OR (95\% CI): $1.34(1.03-1.74) ; P=0.032)$.

\section{Discussion}

\section{Main Findings:}

In this study, we found that administration of hCG for triggering ovulation was associated with increased pregnancy rates in IUI with donor sperm in a natural menstrual cycle. When we divided the patients into subgroups according to IUI number, the increased pregnancy rate in the hCG group was only observed in the single IUI subgroup.

\section{Interpretation:}

Previous research has established that the success of IUI depends on various factors, including maternal age, sperm quality, type of subfertility, ovarian stimulation, and the timing of insemination ${ }^{18}$, ${ }^{19}$. Given that spermatozoa and oocytes have only limited survival times, the appropriate timing of IUI relative to ovulation may be one of the most important factors influencing IUI success ${ }^{6}$. Ovulation typically occurs 25-56 $\mathrm{h}$ following the onset of a spontaneous LH surge, whereas ovulation usually occurs $36-48 \mathrm{~h}$ after hCG administration in natural cycles.

Administration of hCG makes clinical prediction of ovulation more accurate; it permits planning for optimized time intervals between ovulation and insemination possible ${ }^{20}$. This increased accuracy can likely help explain the significantly increased pregnancy rates achieved by the hCG group women in the present study. 
When insemination was performed twice, the window of sperm exposure to the oocyte was significantly increased; this maybe help explain the similar clinical pregnancy rates observed for the two groups ${ }^{21}$.

Several studies of ovarian hyperstimulation IUI cycles have demonstrated beneficial effects of hCG administration on IUI pregnancies ${ }^{22-24}$. One study by Taerk et al. (2017) reported that hCG administration significantly increased clinical pregnancy rates compared with monitoring of spontaneous serum LH surge in subfertile patients undergoing controlled ovarian hyperstimulation IUI cycles ${ }^{22}$. Two additional retrospective studies of stimulated IUI have also reported that higher pregnancy rates resulted when hCG was given to trigger ovulation ${ }^{23,24}$.

Few studies have explored association(s) between hCG administration and pregnancy outcomes in natural cycle IUI. Moreover, the few studies addressing this topic have yielded inconsistent results. A Cochrane meta-analysis by Cantineau et al. (2014) reported no difference in pregnancy rates or live birth rates between natural cycle IUI patients timed according to spontaneous LH surge monitoring or hCG triggering 24 . However, note that these reports included a total of only 264 women, and only one study reported live birth rate data.

To date, only one randomized clinical trial has investigated the pregnancy outcome of hCG administration for triggering ovulation in natural cycle IUI ${ }^{9}$. A total of 300 patients were included in that study, of which 197 women used donor sperm. The trial concluded that administration of hCG resulted in decreased ongoing pregnancy rates. Given that the goal of IUI treatment is to achieve a healthy live birth, it is unfortunate that live birth rate data for the two groups was not reported from the trial.

A retrospective cohort study by El Hachem et al. (2017) found no difference in clinical pregnancy or live birth rates between urinary LH monitoring vs hCG-triggered ovulation in natural unstimulated therapeutic donor sperm insemination cycles ${ }^{11}$. The inclusion criteria of that study were strict: only normo-ovulatory women were included. Notably, whereas the average age of the patients in that study was 32, the mean age of patients in our study was 27 years. We suspect that this 5 year age gap may be a major factor underlying the inconsistent conclusions of the two studies.

\section{Strengths and Limitations:}

The current study had several strengths, the foremost of which was its large cohort size. To the best of our knowledge, ours is the largest-to-date study comparing the two major IUI timing methods for natural cycle IUI. Moreover, our study monitored the live birth rate of natural cycle IUI, data which has been reported rarely in previous studies.

Limitations of our study include the use of urinary LH monitoring, which has been associated with increased probability for false-negative results; such results would cause inaccurate timing and thereby likely contribute to decreased pregnancy rates ${ }^{3}$. However, considering its advantages over alternatives (like serum LH testing) such as ease-of-use, non-invasiveness, and low cost, urinary LH monitoring is widely used in the clinic 26, 27. It also bears mention that our clinical pregnancy rate in the spontaneous LH group was $22.73 \%$, a rate comparable to previously published natural cycle IUI success rates. Second, our study is based on a retrospective design. The inclusion criteria were strict, with only normo-ovulatory women aged [?] 35 years who underwent IUI with high-quality donor sperm included, which enabled analysis of a well-defined cohort of women with no obvious confounding factors.

\section{Conclusion :}

In conclusion, the administration of hCG for triggering of ovulation is associated with significantly higher pregnancy rates compared with spontaneous ovulation in patients undergoing natural IUI. Thus, using hCG for timing ovulation optimizes the chances of success for natural cycle IUI with donor sperm.

\section{Acknowledgments}

We are grateful to Hong-bin Liu and Wei Zhou of Shandong Provincial Hospital Affiliated to Shandong University for technical support. We especially thank all the patients for participating in this study. 


\section{Disclosure of interests}

The author(s) declared no potential conflicts of interest with respect to the research, authorship, and/or publication of this article.

\section{Contribution to authorship}

Conceptualisation was by SM, W-ZJ and W-JP; data curation was by SY and F-HR; formal analysis was by G-QQ, XJ, CW and W-ZJ; and writing was by W-JP, W-ZJ and SM

\section{Details of ethics approval}

The Institutional Review Board, Shandong Provincial Hospital Affiliated to Shandong University, Jinan, China, gave the ethical approval for the study (Number 6166).

\section{Funding:}

The authors disclose receipt of the following financial support for the research, authorship and/or publication of this article: this study was supported by the Key Research and Development Program of Shandong Province (2019GSF108090); the National Natural Science Foundation of China (81801476); the Natural Science Foundation of Shandong Province (ZR2019PH054, ZR2017BH046); the Medical and Health Science and Technology Development Plan of Shandong Province (2016WS0411, 2016WS0404) and the Jinan Science and Technology Development Program (201805026).

\section{References}

1. De Geyter C, Calhaz-Jorge C, Kupka MS, Wyns C, Mocanu E, Motrenko T, et al. ART in Europe, 2014: results generated from European registries by ESHRE: The European IVF-monitoring Consortium (EIM) for the European Society of Human Reproduction and Embryology (ESHRE), 2018. Human reproduction 2018;33: 1586-601.

2. Veltman-Verhulst SM, Hughes E, Ayeleke RO, Cohlen BJ. Intra-uterine insemination for unexplained subfertility, 2016. The Cochrane database of systematic reviews 2016;19: 2 (CD001838-CD).

3. Cohlen B, Bijkerk A, Van der Poel S, Ombelet W. IUI: review and systematic assessment of the evidence that supports global recommendations, 2018. Human reproduction update 2018;24:300-19.

4. Group EC W. Intrauterine insemination, 2009. Human reproduction update 2009;15:265-77.

5. Plosker SM, Jacobson W, Amato P. Predicting and optimizing success in an intra-uterine insemination programme, 1994. Human reproduction 1994;9: 2014-21.

6. Ragni G, Somigliana E, Vegetti W. Timing of intrauterine insemination: where are we? 2004. Fertility and sterility 2004;82:25-35.

7. Evans J, Salamonsen LA. Too much of a good thing? Experimental evidence suggests prolonged exposure to hCG is detrimental to endometrial receptivity, 2013. Human reproduction 2013;28:1610-9.

8. Ezoe K, Daikoku T, Yabuuchi A, Murata N, Kawano H, Abe T, et al. Ovarian stimulation using human chorionic gonadotrophin impairs blastocyst implantation and decidualization by altering ovarian hormone levels and downstream signaling in mice, 2014. Molecular human reproduction 2014;20:1101-16.

9. Kyrou D, Kolibianakis EM, Fatemi HM, Grimbizis GF, Theodoridis TD, Camus M, et al. Spontaneous triggering of ovulation versus HCG administration in patients undergoing IUI: a prospective randomized study, 2012. Reproductive biomedicine online 2012;25:278-83.

10. Kosmas IP, Tatsioni A, Fatemi HM, Kolibianakis EM, Tournaye H, Devroey P. Human chorionic gonadotropin administration vs. luteinizing monitoring for intrauterine insemination timing, after administration of clomiphene citrate: a meta-analysis, 2007. Fertility and sterility 2007;87:607-12. 
11. El Hachem H, Antaki R, Sylvestre C, Lapensee L, Legendre G, Bouet PE. Timing therapeutic donor inseminations in natural cycles: human chorionic gonadotrophin administration versus urinary LH monitoring, 2017. Reproductive biomedicine online 2017;35:174-9.

12. Martinez AR, Bernadus RE, Voorhorst FJ, Vermeiden JP, Schoemaker J. A controlled study of human chorionic gonadotrophin induced ovulation versus urinary luteinizing hormone surge for timing of intrauterine insemination, 1991. Human reproduction (Oxford, England) 1991;6:1247-51.

13. Mohamad E. Ghanem N, Bakre, Mohamad A. Emam, Laila A. The effects of timing of intrauterine insemination in relation to ovulation and the number of inseminations on cycle pregnancy rate in common infertility etiologies, 2011. Human reproduction 2011. 26, 576-83

14. Rijsdijk OE, Cantineau AE, Bourdrez P, Gijsen TP, Gondrie ET, Sprengers O, et al. Intrauterine insemination: simultaneous with or $36 \mathrm{~h}$ after HCG? A randomized clinical trial, 2019. Reproductive biomedicine online 2019;39, 262-268

15. Blockeel C, Knez J, Polyzos NP, De Vos M, Camus M, Tournaye H. Should an intrauterine insemination with donor semen be performed 1 or 2 days after the spontaneous LH rise? 2014. A prospective RCT. Human reproduction 2014; 29:697 -703.

16. Cooper TG, Noonan E, von Eckardstein S, Auger J, Baker HW, Behre HM, et al. World Health Organization reference values for human semen characteristics, 2010. Hum Reprod Update 2010; 16:231-45.

17. Zarek SM, Hill MJ, Richter KS, Wu M, DeCherney AH, Osheroff JE, et al. Single-donor and double-donor sperm intrauterine insemination cycles: does double intrauterine insemination increase clinical pregnancy rates? 2014. Fertility and sterility 2014;102:739-43.

18. Iberico G, Vioque J, Ariza N, Lozano JM, Roca M, Llacer J, et al. Analysis of factors influencing pregnancy rates in homologous intrauterine insemination, 2004. Fertility and sterility 2004;81(5):1308-13.

19. Thijssen A, Creemers A, Van der Elst W, Creemers E, Vandormael E, Dhont N, et al. Predictive factors influencing pregnancy rates after intrauterine insemination with frozen donor semen: a prospective cohort study, 2017. Reproductive biomedicine online 2017;34:590-7.

20. ESHRE Capri Workshop Group. Intrauterine insemination, 2009. Hum Reprod Update 2009; 15:265-77.

21. Arab-Zozani, M. and C. O. Nastri. Single versus double intrauterine insemination (IUI) for pregnancy: A systematic review and meta-analysis, 2017. Eur J Obstet Gynecol Reprod Biol 2017; 215: 75-84.

22. Taerk E, Hughes E, Greenberg C, Neal M, Amin S, Faghih M, et al. Controlled Ovarian Hyperstimulation with Intrauterine Insemination Is More Successful After r-hCG Administration Than Spontaneous LH Surge, 2017. Journal of reproduction \& infertility 2017;18:316-22.

23. Mitwally MF, Abdel-Razeq S, Casper RF. Human chorionic gonadotropin administration is associated with high pregnancy rates during ovarian stimulation and timed intercourse or intrauterine insemination, 2004. Reproductive biology and endocrinology 2004;2:55.

24. Jarvela IY, Tapanainen JS, Martikainen H. Improved pregnancy rate with administration of hCG after intrauterine insemination: a pilot study, 2010. Reproductive biology and endocrinology 2010;8:18.

25. Cantineau AE, Janssen MJ, Cohlen BJ , Allersma T. Synchronised approach for intrauterine insemination in subfertile couples, 2014. The Cochrane database of systematic reviews 2014:Cd006942.

26. Miller PB, Soules MR. The usefulness of a urinary LH kit for ovulation prediction during menstrual cycles of normal women, 1996. Obstet Gynecol 1996; 87:13-7.d

27. Lemmens L, Kos S, Beijer C, Braat DDM, Nelen WLDM, Wetzels AMM. Techniques used for IUI: is it time for a change? 2017. Hum Reprod 2017;32:1835-45.

Table 1. Demographic characteristics of the patient population. 


\begin{tabular}{llll}
\hline Characteristics & HCG administration & Spontaneous ovulation & $P$ \\
\hline Patients, $\mathrm{n}$ & 4343 & 1267 & \\
Age $($ years $)$ & $27.25 \pm 3.40$ & $27.51 \pm 3.29$ & 0.014 \\
BMI $\left(\mathrm{kg} / \mathrm{m}^{2}\right)$ & $22.11 \pm 3.15$ & $22.14 \pm 3.19$ & 0.864 \\
AMH $(\mathrm{ng} / \mathrm{ml})$ & $4.20 \pm 2.90$ & $4.28 \pm 2.93$ & 0.54 \\
FSH $(\mathrm{IU} / \mathrm{L})$ & $6.64 \pm 1.77$ & $6.83 \pm 1.82$ & 0.001 \\
LH $(\mathrm{mIU} / \mathrm{ml})$ & $4.88 \pm 2.39$ & $5.07 \pm 2.16$ & 0.012 \\
Estradiol $(\mathrm{pg} / \mathrm{mL})$ & $34.75 \pm 13.17$ & $34.25 \pm 12.69$ & 0.617 \\
Endometrium thickness & $1.01 \pm 0.11$ & $1.02 \pm 0.14$ & 0.06 \\
on the day of & & & \\
insemination $(\mathrm{cm})$ & & & \\
IUI number & $1.89 \pm 0.31$ & & $<0.001$ \\
\hline
\end{tabular}

Data are presented as mean $\pm \mathrm{SD}$ for continuous variables

$\mathrm{BMI}=$ body mass index $; \mathrm{AMH}=$ anti-Müllerian hormone; IUI $=$ intrauterine insemination.

Table 2. Reproductive outcomes of the patient population.

\begin{tabular}{|c|c|c|c|c|c|c|}
\hline Characteristic & $\begin{array}{l}\text { HCG } \\
\text { adminis- } \\
\text { tration }\end{array}$ & $\begin{array}{l}\text { Spontaneous } \\
\text { ovulation }\end{array}$ & $\begin{array}{l}\text { OR }(95 \% \\
\text { CI })\end{array}$ & $\begin{array}{l}\text { AOR }(95 \% \\
\text { CI })\end{array}$ & $\mathrm{P}$ & $P$ adj \\
\hline $\begin{array}{l}\text { Clinical } \\
\text { pregnancy }\end{array}$ & $\begin{array}{l}1059 / 3879 \\
(27.30 \%)\end{array}$ & $\begin{array}{l}92 / 368 \\
(25.00 \%)\end{array}$ & $1.12(0.881 .44)$ & $1.12(0.881 .44)$ & 0.343 & 0.356 \\
\hline Miscarriage & $\begin{array}{l}110 / 1059 \\
(10.39 \%)\end{array}$ & $\begin{array}{l}16 / 92 \\
(17.39 \%)\end{array}$ & $0.55\left(\begin{array}{lll}0.31 & 0.99\end{array}\right)$ & $0.52\left(\begin{array}{lll}0.29 & 0.94)\end{array}\right.$ & 0.039 & 0.029 \\
\hline Live birth & $\begin{array}{l}949 / 3879 \\
(24.46 \%)\end{array}$ & $\begin{array}{l}76 / 368 \\
(20.66 \%)\end{array}$ & $1.24\left(\begin{array}{lll}0.96 & 1.62)\end{array}\right.$ & $1.25(0.961 .62)$ & 0.102 & 0.103 \\
\hline
\end{tabular}

Data are presented as n/n (\%) for dichotomous variables OR Odd ratio, AOR adjusted odd ratio,

Clinical pregnancies were confirmed by the presence of a fetal heartbeat on ultrasound scanning 7 weeks after insemination. Miscarriage was defined as loss of a clinical pregnancy before the 28th week of gestation. Live birth was defined as delivery of a live neonate after 28 weeks' gestation.

Analyses were adjusted for maternal age, BMI, number of intrauterine insemination events, and basal hormone levels (FSH, LH, estradiol, AMH)

Table 3. Reproductive outcomes of the single IUI group.

\begin{tabular}{lllllll}
\hline & $\begin{array}{l}\text { HCG } \\
\text { adminis- }\end{array}$ & $\begin{array}{l}\text { Spontaneous } \\
\text { ovulation }\end{array}$ & $\begin{array}{l}\text { OR }(\mathbf{9 5 \%} \\
\text { CI) }\end{array}$ & $\begin{array}{l}\text { AOR }(\mathbf{9 5 \%} \\
\text { CI) }\end{array}$ & P & P adj \\
\hline Characteristics tration & $1059 / 3879$ & $92 / 368$ & $1.12(0.881 .44)$ & $1.12(0.881 .44)$ & 0.343 & 0.356 \\
pregnancy & $(27.30 \%)$ & $(25.00 \%)$ & & & & 0.029 \\
Miscarriage & $110 / 1059$ & $16 / 92$ & $0.55(0.310 .99)$ & $0.52(0.290 .94)$ & 0.039 & 0.103 \\
& $(10.39 \%)$ & $(17.39 \%)$ & & & & \\
Live birth & $949 / 3879$ & $76 / 368$ & $1.24(0.961 .62)$ & $1.25(0.961 .62)$ & 0.102 & \\
& $(24.46 \%)$ & $(20.66 \%)$ & & & & \\
\hline
\end{tabular}


Data are presented as n/n (\%) for dichotomous variables OR Odd ratio, AOR adjusted odd ratio,

Clinical pregnancies were confirmed by the presence of a fetal heartbeat on ultrasound scanning 7 weeks after insemination. Miscarriage was defined as loss of a clinical pregnancy before the 28 th week of gestation. Live birth was defined as delivery of a live neonate after 28 weeks' gestation.

Analyses were adjusted for maternal age, BMI, number of intrauterine insemination events, and basal hormone levels (FSH, LH, estradiol, AMH)

Table 4. Reproductive outcomes of the double IUI group.

\begin{tabular}{lllllll}
\hline & $\begin{array}{l}\text { HCG } \\
\text { adminis- } \\
\text { Characteristics tration }\end{array}$ & $\begin{array}{l}\text { Spontaneous } \\
\text { ovulation }\end{array}$ & $\begin{array}{l}\text { OR }(\mathbf{9 5 \%}) \\
\text { CI) }\end{array}$ & $\begin{array}{l}\text { AOR }(\mathbf{9 5 \%} \\
\text { CI) }\end{array}$ & P & P adj \\
\hline $\begin{array}{l}\text { Clinical } \\
\text { pregnancy }\end{array}$ & $1059 / 3879$ & $92 / 368$ & $1.12(0.881 .44)$ & $1.12(0.881 .44)$ & 0.343 & 0.356 \\
Miscarriage & $(27.30 \%)$ & $(25.00 \%)$ & & & & 0.029 \\
& $110 / 1059$ & $16 / 92$ & $0.55(0.310 .99)$ & $0.52(0.290 .94)$ & 0.039 & 0.103 \\
Live birth & $(10.39 \%)$ & $(17.39 \%)$ & & & & \\
& $949 / 3879$ & $76 / 368$ & $1.24(0.961 .62)$ & $1.25(0.961 .62)$ & 0.102 & \\
& $(24.46 \%)$ & $(20.66 \%)$ & & & & \\
\hline
\end{tabular}

Data are presented as n/n (\%) for dichotomous variables OR Odd ratio, AOR adjusted odd ratio

Clinical pregnancies were confirmed by the presence of a fetal heartbeat on ultrasound scanning 7 weeks after insemination. Miscarriage was defined as loss of a clinical pregnancy before the 28th week of gestation. Live birth was defined as delivery of a live neonate after 28 weeks' gestation.

Analyses were adjusted for maternal age, BMI, number of intrauterine insemination events, and basal hormone levels (FSH, LH, estradiol, AMH) 